\title{
PERTURBATION OF COMPLETE ORTHONORMAL SETS AND EIGENFUNCTION EXPANSIONS
}

\author{
JERRY L. KAZDAN ${ }^{1}$
}

\begin{abstract}
A technique is given for determining the asymptotic properties of vectors which are perturbations of a given basis-the eigenfunctions a selfadjoint operator. Its application is illustrated by a differential equation example, not using the Hilbert space norm. An estimate is also given for the codimension of the span of a perturbed set of vectors.
\end{abstract}

In many specific problems, one has a selfadjoint operator with a complete set of eigenvectors and a second operator differing from it by a "small" operator. The problem is to determine the properties of the second from those of the first. For example, are the eigenvectors of the second operator complete and are they in any sense close to those of the first operator? Although the problem is old, most solutions are quite technically complicated.

There are two general approaches. The first [1], [2] shows that the eigenvectors of the perturbed operator are asymptotically close to those of the unperturbed operator, from which the completeness is deduced. In this approach the asymptotic behavior of the eigenfunctions is the difficult part. Bary-Krein (see [3, p. 265]) and later in a special case Birkhoff-Rota [1] observed that the completeness then follows rather easily. The second method [4], [5] utilizes contour integration around points of the spectrum to establish the results.

In this paper we follow the first approach. It turns out that with a slight abstraction, the asymptotic behavior follows in a brief and elementary way. This is our Theorem 1 . In $\$ 2$ we apply it to classical Sturm-Liouville theory. $\$ 3$ illustrates a concrete application, not in a Hilbert space, that follows from our method but is inaccessible to the more abstract approach of $\S 1$. Although the results are known, our proofs are considerably shorter than previous ones. Moreover, the techniques should be useful in other specific applications where Hilbert space theory is not applicable. In $\$ 4$ we briefly consider more general perturbation of bases and give an amusing estimate of the codimension of the span of the perturbed vectors.

Received by the editors May 28, 1970.

AMS 1969 subject classifications. Primary 4748, 3430, 3453; Secondary 4730, 4760.

Key words and phrases. Eigenfunctions, perturbation, Schauder basis, asymptotic.

1 Supported in part by NSF GP 4503.

Copyright (c) 1971, American Mathematical Society 
1. Let $L_{0}$ be an unbounded selfadjoint operator with domain $D\left(L_{0}\right)$ in a Hilbert space $H$, having simple eigenvalues $\lambda_{k} \rightarrow \infty$ with the corresponding complete set of orthonormal eigenvectors $e_{k}, L_{0} e_{k}$ $=\lambda_{k} e_{k}$.

Let $L=L_{0}+L_{1}$, where $L_{1}$ is a bounded but not necessarily selfadjoint operator with $D(L)=D\left(L_{0}\right) . \mu_{k}$ and $X_{k}$ will denote the eigenvalues and corresponding normalized eigenvectors of $L, L X_{k}=\mu_{k} X_{k}$. The symbol $\delta_{k}=\min _{j \neq k}\left|\lambda_{j}-\lambda_{k}\right|$ is the "isolation distance" of the eigenvalue $\lambda_{k}$. We begin with an easy inequality.

Lemma 1. If $Z \in D\left(L_{0}\right)$ and $Z \perp e_{k}$, then

$$
\left|\left(L_{0}-\lambda_{k}\right) Z\right| \geqq \delta_{k}|Z| \text {. }
$$

Proof. Write $Z=\sum_{1}^{\infty} a_{j} e_{j}$ and $Z_{n}=\sum_{1}^{n} a_{j} e_{j}$. Then, since $a_{k}=0$,

$$
\begin{aligned}
\left|\left(L_{0}-\lambda_{k}\right) Z\right|^{2} & \geqq\left|\left(L_{0}-\lambda_{k}\right) Z_{n}\right|^{2}=\sum_{1}^{n}\left|\lambda_{j}-\lambda_{k}\right|^{2}\left|a_{j}\right|^{2} \\
& \geqq \delta_{k}^{2} \sum_{1}^{n}\left|a_{j}\right|^{2}=\delta_{k}^{2}\left|Z_{n}\right|^{2} .
\end{aligned}
$$

Now let $n \rightarrow \infty$ on the right.

The following lemma shows that under certain conditions $X_{k} \rightarrow e_{k}$ as $k \rightarrow \infty$.

LEMMa 2. $\left|e_{k}-X_{k}\right| \leqq 2\left(\left|\lambda_{k}-\mu_{k}\right|+\left|L_{1}\right|\right) / \delta_{k}$.

Proof. Since $L X_{k}=\mu_{k} X_{k}$, then $\left(L_{0}-\lambda_{k}\right) X_{k}=\left(\mu_{k}-\lambda_{k}\right) X_{k}-L_{1} X_{k}$. We always can write $X_{k}=a e_{k}+Z_{k}$, where $a$ is a scalar (which we can assume is $\geqq 0$ by multiplying $X_{k}$ by a scalar of absolute value one), and where $Z_{k}$ is orthogonal to the nullspace of $L_{0}-\lambda_{k}$, that is, to $e_{k}$. Now $Z_{k}$ satisfies the inhomogeneous equation $\left(L_{0}-\lambda_{k}\right) Z_{k}$ $=\left(\mu_{k}-\lambda_{k}\right) X_{k}-L_{1} X_{k}$ so that by Lemma 1 ,

$$
\delta_{k}\left|Z_{k}\right| \leqq\left|\left(\mu_{k}-\lambda_{k}\right) X_{k}-L_{1} X_{k}\right| \leqq\left|\mu_{k}-\lambda_{k}\right|+\left|L_{1}\right| \text {. }
$$

Also, $1=\left|X_{k}\right|^{2}=\left|a e_{k}\right|^{2}+\left|Z_{k}\right|^{2}$, so that $0 \leqq 1-a \leqq\left|Z_{k}\right|$. Consequently,

$$
\begin{aligned}
\left|e_{k}-X_{k}\right| & \leqq\left|(1-a) e_{k}-Z_{k}\right| \leqq 1-a+\left|Z_{k}\right| \\
& \leqq 2\left|Z_{k}\right| \leqq 2 \frac{\left|\mu_{k}-\lambda_{k}\right|+\left|L_{1}\right|}{\delta_{k}} .
\end{aligned}
$$

With but minor modifications, both Lemma 1 and Lemma 2 remain valid if the eigenvalue $\lambda_{k}$ is not simple. However, that assumption 
becomes more important when one wants to prove the completeness of the $X_{k}$ 's. The key is

LEMMA 3 (BARY-KREIN). Let $\left\{e_{k}\right\}$ be an orthonormal basis for $H$ and $X_{k}$ a set of linearly independent vectors in the sense that $\sum a_{k} X_{k}=0$ strongly implies $a_{k}=0$. If $\sum\left|X_{k}-e_{k}\right|^{2}<\infty$ then the set $\left\{X_{k}\right\}$ is a Schauder basis for $H$.

REMARK. If the $X_{j}^{\prime}$ 's are not linearly independent, one can still estimate the codimension of their span. See $\$ 4$.

Our theorem now follows immediately from these lemmas.

TheOREM 1. Let $L_{0} e_{k}=\lambda_{k} e_{k}$ and $L X_{k}=\mu_{k} X_{k}$, where $L=L_{0}+L_{1}$ with $L_{0}$ selfadjoint and having pure simple point spectrum. If (a) $L_{1}$ is bounded, (b) $\left|\lambda_{k}-\mu_{k}\right|<\alpha$, and (c) $\sum 1 / \delta_{k}^{2}<\infty$, then (i) $X_{k}=e_{k}$ $+O\left(1 / \delta_{k}\right)$ as $k \rightarrow \infty$, and (ii) the eigenvectors $\left\{X_{k}\right\}$ of $L$ are complete.

2. It is straightforward to apply the above to classical SturmLiouville theory. Moreover, an examination of the above proofs shows that one can ignore the delicate issues involved in dealing with strict selfadjoint extensions of unbounded operators. For example, consider

$$
L_{0} u=-u^{\prime \prime}, \quad L u=-u^{\prime \prime}+q u,
$$

where $q \in C[0,1]$ is real, and say the boundary conditions are $u(0)=u(1)=0$. Then $L_{0}$ acting on $B C^{2}=\left\{u \in C^{2}[0,1] \mid u(0)=u(1)=0\right\}$ is formally selfadjoint (by closing the operator it becomes strictly selfadjoint, but we ignore that). Its eigenvalues and eigenfunctions are $\lambda_{n}=n^{2} \pi^{2}, e_{n}(x)=2^{1 / 2} \sin n \pi x, n=1,2, \cdots$.

By Sturm's oscillation theorem, $L$ also acting on $B C^{2}$ has an infinite number of eigenvalues $\mu_{n}$ and $\left|\mu_{n}-\lambda_{n}\right| \leqq|q|_{\infty}\left(||_{\infty} \equiv\right.$ uniform norm on $[0,1])$. Since $\delta_{n}=\min _{j \neq n}\left|j^{2} \pi^{2}-n^{2} \pi^{2}\right|=(2 n-1) \pi^{2}$, we conclude that the eigenfunctions $u_{n}$ of $L$ have the asymptotic behavior $u_{n}(x)=2^{1 / 2} \sin n \pi x+O(1 / n)$ in $L_{2}[0,1]$ as $n \rightarrow \infty$, and that the $u_{n}$ 's are complete in $L_{2}[0,1]$. Note that since $L$ is formally selfadjoint the $u_{n}$ 's are orthonormal so the special case of Lemma 3 proved in [1] is adequate. By standard methods one can show that if $f \in B C^{2}$ (or even if $f^{\prime} \in L_{2}[0,1]$ and satisfies the boundary conditions) then its eigenfunction expansion converges uniformly.

3. Our abstract method can of ten be adapted to concrete situations where $L_{0}$ and $L=L_{0}+L_{1}$ have different domains. For variety, we shall use uniform norms in place of Hilbert space norms in the following illustration. 
Consider the operator $L_{0} u=-u^{\prime \prime}$ acting on $B C^{2}$, as before, and the perturbed operator $L u=-u^{\prime \prime}+q u$, acting on $C^{2}[0,1]$ functions but with the boundary values

$$
u(0)+c_{1} u^{\prime}(0)=0, \quad u(1)+c_{2} u^{\prime}(1)=0,
$$

where for simplicity, we assume that $c_{1} \neq 0, c_{2} \neq 0$. The idea is to replace the estimate of Lemma 1 by a similar one using variation of parameters and then use this estimate in imitating the proof of Lemma 2.

THEOREM 2. $u_{n}(x)=2^{1 / 2} \cos n \pi x+O(1 / n)$ uniformly as $n \rightarrow \infty$ and the $u_{n}$ 's are complete in $L_{2}[0,1]$.

Proof. Let $\phi_{n}(x)=2^{1 / 2} \cos n \pi x, \psi_{n}(x)=2^{1 / 2} \sin n \pi x$, and let $u_{n}$ be the normalized $n$th eigenfunction of $L$ with corresponding eigenvalue $\mu_{n}$. Then $L u_{n}=\mu_{n} u_{n}$ so that $u_{n}^{\prime \prime}+n^{2} \pi^{2} u_{n}=\left(n^{2} \pi^{2}-\mu_{n}+q\right) u_{n}$. By using variation of parameters this can be written as the integral equation $u_{n}=a_{n} \phi_{n}+b_{n} \psi_{n}+z_{n}$, where

$$
z_{n}(x)=\frac{1}{n \pi} \int_{0}^{x}\left[n^{2} \pi^{2}-\mu_{n}+q(t)\right] u_{n}(t) \sin n \pi(x-t) d t,
$$

and where the constants $a_{n}$ and $b_{n}$ are determined by the boundary values of $u$. As before, we multiply $u_{n}$ by a scalar of absolute value one to insure that $a_{n} \geqq 0$.

Note that by the Schwarz inequality (here $\|_{2}$ is $L_{2}(0,1)$ norm)

$$
\left|z_{n}\right|_{2} \leqq\left|z_{n}\right|_{\infty} \leqq \frac{\left|n^{2} \pi^{2}-\mu_{n}\right|+|q|_{2}}{n \pi} \leqq \frac{\alpha}{n},
$$

since under our hypothesis $\left|n^{2} \pi^{2}-\mu_{n}\right| \leqq$ constant independent of $n$, as can be proved by the Sturm oscillation theorem. Also note that $u_{n}(0)=a_{n} 2^{1 / 2}$ and $u_{n}^{\prime}(0)=n \pi b_{n} 2^{1 / 2}$.

Claim. As $n \rightarrow \infty\left|a_{n}-1\right|=O(1 / n)$ and $\left|b_{n}\right|=O(1 / n)$.

This is proved as follows. Now $0=u_{n}(0)+c_{1} u_{n}^{\prime}(0)=\left(a_{n}+c_{1} n \pi b_{n}\right) 2^{1 / 2}$, so that $\left|a_{n}\right|=\left|c_{1} n \pi b_{n}\right|$. But

$$
a_{n}=\left|a_{n} \phi_{n}\right|_{2}=\left|u_{n}-b_{n} \psi_{n}-z_{n}\right|_{2} \leqq 1+\left|b_{n}\right|+\left|z_{n}\right|_{2},
$$

and similarly $1 \leqq a_{n}+\left|b_{n}\right|+\left|z_{n}\right|_{2}$. Putting these two lines together we obtain the claimed orders of growth. These inequalities show that

$$
\left|u_{n}-\phi_{n}\right|_{\infty}=\left|\left(a_{n}-1\right) \phi_{n}+b_{n} \psi_{n}+z_{n}\right|_{\infty}=O(1 / n) .
$$

This proves the asymptotic result of Theorem 2. The completeness again follows from Lemma 3. 
4. Let $\left\{e_{j}\right\}$ be a complete orthonormal set for a separable Hilbert space. If the vectors $\left\{x_{j}\right\}$ are close to the $\left\{e_{j}\right\}$, we estimate the codimension of the span of $\left\{x_{j}\right\}$. This extends Lemma 3. $R(L)$ and $N(L)$ denote the range and nullspace of $L$.

Lemma 4. Let $P$ be an orthogonal projector. Then

$$
\operatorname{dim} R(P)=\sum\left|P e_{j}\right|^{2} .
$$

Proof. The right side is just the trace of $P$. In more detail, let $\left\{\epsilon_{j}\right\}$ be an orthonormal basis for $R(P)$. Then

$$
\begin{aligned}
\sum_{j}\left|P e_{j}\right|^{2} & =\sum_{j} \sum_{k}\left|\left\langle e_{j}, \epsilon_{k}\right\rangle\right|^{2}=\sum_{k} \sum_{j}\left|\left\langle e_{j}, \epsilon_{k}\right\rangle\right|^{2} \\
& =\sum_{k}\left|\epsilon_{k}\right|^{2}=\operatorname{dim} R(P) .
\end{aligned}
$$

THEOREM 3. Let $L$ be a linear map admitting an adjoint $L^{*}$. If $\left\{x_{j}\right\}$ $\in R(L)$ then

$$
\operatorname{dim} R(L)^{\perp} \leqq \sum\left|x_{j}-e_{j}\right|^{2} .
$$

In particular, if $S$ is any subspace, then for any vectors $\left\{x_{j}\right\} \in \mathcal{S}$ we have (by letting $L=$ projection into $s$ )

$$
\operatorname{dim} \mathcal{S}^{\perp} \leqq \sum\left|x_{j}-e_{j}\right|^{2}
$$

Proof. Let $P$ denote the projector into $N\left(L^{*}\right)$. Now $x_{j} \perp N\left(L^{*}\right)$ so

$$
\left|x_{j}-e_{j}\right|^{2}=\left|P\left(x_{j}-e_{j}\right)\right|^{2}+\left|P^{\perp}\left(x_{j}-e_{j}\right)\right|^{2} \geqq\left|P e_{j}\right|^{2} \text {. }
$$

Summing over $j$ and applying Lemma 4 , we conclude that $\operatorname{dim} N\left(L^{*}\right)$ $\leqq \sum\left|x_{j}-e_{j}\right|^{2}$. But $N\left(L^{*}\right)=R(L)^{\perp}$.

\section{BIBLIOGRAPHY}

1. G. Birkhoff and G.-C. Rota, Ordinary differential equations. Introduction to higher mathematics, Ginn, Boston, Mass., 1962, Chapter 11. MR 25 \#2253.

2. E. Ince, Ordinary differential equations, reprint, Dover, New York, 1944. MR 6,65 .

3. T. Kato, Perturbation theory for linear operators, Die Grundlehren der math. Wissenschaften, Band 132, Springer-Verlag, New York, 1967. MR 34 \#3324.

4. J. Schwartz, Perturbations of spectral operators, and applications. I: Bounded perturbations, Pacific J. Math. 4 (1954), 415-458. MR 16, 144.

5. E. Titchmarsh, Eigenfunction expansions associated with second-order differential equations, Clarendon Press, Oxford, 1946. MR 8, 458.

University of Pennsylvania, Philadelphia, Pennsylvania 19104 